\title{
Primeiro registro de ocorrência de Hemerobius gaitoi Monserrat, 1996 e Hemerobius hernandezi Monserrat, 1996 (Neuroptera: Hemerobiidae) para o estado de Minas Gerais, Brasil
}

\section{First report of the occurrence of Hemerobius gaitoi Monserrat, 1996 and Hemerobius hernandezi Monserrat, 1996 (Neuroptera: Hemerobiidae) for the state of Minas Gerais, Brazil}

Marise Silva ${ }^{*}$, Rogéria Inês Rosa Lara², Brígida Souza'

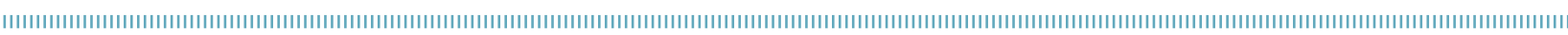

RESUMO: O presente estudo relata, pela primeira vez, a ocorrência de Hemerobius gaitoi Monserrat, 1996 e Hemerobius hernandezi Monserrat, 1996 (Neuroptera: Hemerobiidae) para o estado de Minas Gerais, Brasil.

PALAVRAS-CHAVE: Brasil; Hemerobiidae; Hemerobius gaitoi; Hemerobius hernandezi; predador.

\begin{abstract}
The present study reports for the first time the occurrence of Hemerobius gaitoi Monserrat, 1996 and Hemerobius hernandezi Monserrat, 1996 (Neuroptera: Hemerobiidae) in the state of Minas Gerais, Brazil.
\end{abstract}

KEYWORDS: Brazil; Hemerobiidae; Hemerobius gaitoi; Hemerobius hernandezi; predator. 
Dentre os hemerobiídeos, Hemerobius Linnaeus, 1758 é o gênero com maior riqueza de espécies, com cerca de 150 espécies descritas, distribuídas em todos os continentes, exceto na Antártida e em algumas ilhas dos oceanos Índico, Atlântico e Pacífico (Monserrat, 2002). Para as Américas Central e do Sul são registradas 30 espécies (Monserrat, 2002); para o Brasil são 5: Hemerobius lentus Hagen, 1861, Hemerobius bolivari Banks, 1910, Hemerobius frontalis Navás, 1932, Hemerobius gaitoi Monserrat, 1996 e Hemerobius hernandezi Monserrat, 1996 (Monserrat, 1990, 1996, 1998).

H. gaitoi é amplamente distribuída nos trópicos: México, Guatemala, Costa Rica, República Dominicana, Venezuela, Equador e Brasil (Monserrat, 2002); para o Brasil há relatos de ocorrência para os estados de São Paulo (Lara; Freitas, 2003) e Rio Grande do Sul (Monserrat, 1998). $H$. hernandezi tem registros para o México, Guatemala, Nicarágua, Costa Rica, Panamá, Colômbia, Venezuela, Brasil (Rio Grande do Sul) e Paraguai (Monserrat, 1998, 2002).

O reconhecimento de $H$. gaitoi é realizado, principalmente, pelas características de coloração de suas asas anteriores que é amarelo-palha com duas manchas marrom-escuras na regiáo basal e da genitália do macho que apresenta ectoprocto alongado, arredondado posteriormente e margem interna com projeção digitiforme apicalmente e cinco a oito cerdas modificadas, largas basalmente e de extremidade fina; gonarco delgado com placas laterais ovoides, alargadas, margem dorsal em forma de "U" aberto e membrana acima dessa margem com numerosas gonosetas; entoprocessos robustos, curtos, com extremidade apical aguda, margem basal larga em vista lateral, curvados para baixo em sua porção mediana (Monserrat, 1996, 2002).

H. hernandezi apresenta asas anteriores ovais, castanho-claras, venação e pigmentaçáo marrom-escura em todas as suas regióes e espaço costal expandido basalmente; a genitália do macho tem ectoprocto alongado em vista lateral, robusto em vista dorsal, margem dorsal levemente convexa, margem ventral lobulada basalmente, margem interna com projeção digitiforme subcônica apicalmente, curvada para dentro e internamente há várias cerdas longas, levemente lanceoladas; gonarco delgado, placas laterais ovoides, alargadas, margem dorsal em forma de " $U$ " aberto; entoprocessos subcônicos, quase paralelos em vista posterior, levemente arqueados em vista lateral (MONSERRAT, 1996, 2002).

As amostragens foram efetuadas quinzenalmente entre março de 2010 e fevereiro de 2011 em um fragmento de vegetação nativa, na Mata do Baú, município de Barroso,

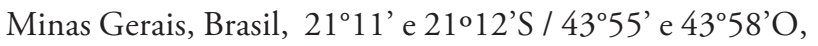
altitude 900-1250 m (NApOleấo, 1979). São aproximadamente 400 ha com formaçôes vegetacionais caracterizadas por floresta semidecidual montana, floresta ripária e campo cerrado (Menini-Neto et al., 2004). O clima da regiâo é tropical de altitude, caracterizado por verôes quentes e chuvosos e invernos secos e frios (clima Cwbmesotérmico de Koppen).

As amostragens foram realizadas em áreas de florestas semidecidual e ripária com o uso de rede entomológica de $30 \mathrm{~cm}$ de diâmetro, haste de 1,5 m de comprimento e confeccionada com tecido voil. Durante as coletas realizadas ao longo de trilhas de aproximadamente $1 \mathrm{~m}$ de largura, existentes no interior da mata, procedia-se a batida da rede entomológica nos galhos e folhas da vegetação, de forma aleatória, e capturavam-se os exemplares a medida em que eles se deslocavam; foram realizadas quinzenalmente duas horas de varredura em cada tipo de vegetação. O material foi preservado em álcool etílico a $70 \%$ e identificados segundo Monserrat (1996). Os espécimes estudados foram depositados na Coleção Entomológica do Departamento de Entomologia da Universidade Federal de Lavras (UFLA), Lavras, Minas Gerais, Brasil.

Dos nove indivíduos de H. gaitoi (Fig. 1) coletados, quatro foram obtidos na floresta semidecidual (entre outubro de 2010 e janeiro de 2011) e cinco na na floresta ripária (em outubro e dezembro de 2010). Dos quatro indivíduos de H. hernandezi (Fig. 2), três foram coletados na floresta semidecidual (em agosto, outubro e novembro de 2010) e um na floresta ripária (dezembro de 2010). As duas espécies têm registros de coletas em áreas de florestas montanhosas e úmidas (Monserrat, 2002). A partir de dados de material

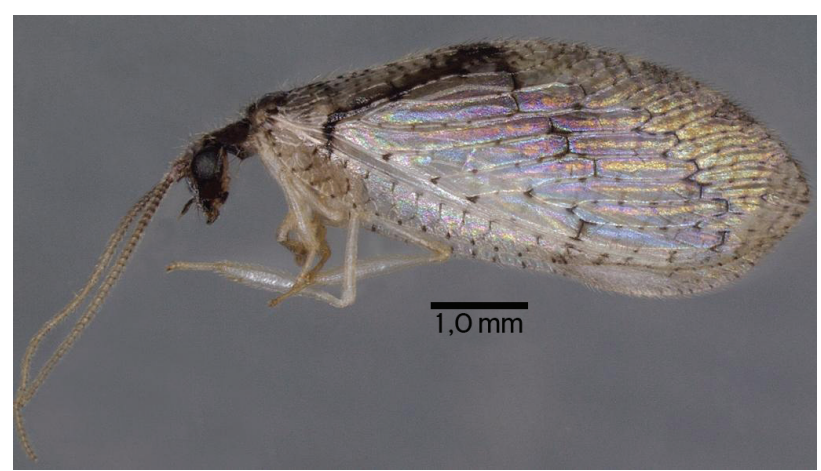

Figura 1. Hemerobius gaitoi Monserrat, 1996 coletado na Mata do Baú, Barroso, Minas Gerais, Brasil.

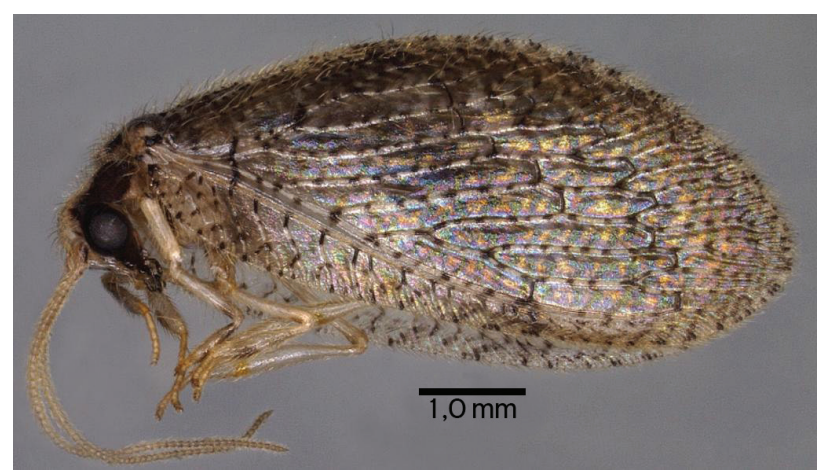

Figura 2. Hemerobius hernandezi Monserrat, 1996 coletado na Mata do Baú, Barroso, Minas Gerais, Brasil. 
estudado para descrição das espécies (MonserRat, 1996) e da fauna da Costa Rica (Monserrat, 2002), H. hernandezi ocorreu em todos os meses do ano e o período de voo dos adultos de $H$. gaitoi ocorreu entre janeiro e abril e de junho a agosto. Os resultados obtidos indicaram que $H$. gaitoi pode ser obtido também na primavera (outubro e novembro) e no início do verão (dezembro).

Quanto à biologia dessas duas espécies, foram observados vestígios de pequenos artrópodes no intestino de $H$. gaitoi e de $H$. hernandezi, para a primeira também foram encontrados pólen e esporos; ambas espécies apresentam grande amplitude altitudinal, e para $H$. hernandezi há registros de captura de indivíduos acima de $2000 \mathrm{~m}$ (Monserrat, 1996).
Estes sáo os primeiros relatos de ocorrência de $H$. gaitoi e de $H$. hernandezi no estado de Minas Gerais, o que amplia o conhecimento da distribuição geográfica dessas duas espécies de hemerobiídeos para o Brasil.

\section{AGRADECIMENTOS}

Ao Conselho Nacional de Desenvolvimento Científico e Tecnológico (CNPq) pela bolsa de mestrado e à Fundação de Amparo à Pesquisa do Estado de Minas Gerais (FAPEMIG) pelo auxílio financeiro para condução do projeto inserido no Programa Biota Minas.

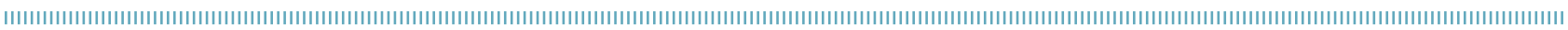

\section{REFERÊNCIAS}

LARA, R.I.R.; FREITAS, S. Caracterização morfológica de espécies de Hemerobius Linnaeus, 1758 (Neuroptera, Hemerobiidae) associadas a cultivo de café (Coffea arabica L.), milho (Zea mays L.) e erva-mate (Ilex paraguariensis St. Hill.). Revista Brasileira de Entomologia, v.47, p.427-434, 2003.

MENINI NETO, L.; ASSIS, L.C.S.; FORZZA, R.C. A família Orchidaceae em um fragmento de floresta estacional semidecidual, no município de Barroso, Minas Gerais, Brasil. Lundiana, v.4, n. 1, p.9-27, 2004.

MONSERRAT, V.J. A systematic checklist of the Hemerobiidae of the world (Insecta: Neuroptera). In: MANSEL, M.W.; ASPOCK, $\mathrm{H}$. Advances in Neuropterology. Pretoria: Proceedings of the Third International Symposium on Neuropterology, 1990. v.3, p.215-262.
MONSERRAT, V.J. Revisión del genero Hemerobius de Latinoamerica (Neuroptera, Hemerobiidae). Fragmenta Entomologica, v.27, n.2, p.399-523, 1996.

MONSERRAT, V.J. Nuevos datos sobre los hemeróbidos de América (Neuroptera: Hemerobiidae). Journal of Neuropterology, v.1, p.109-153, 1998.

MONSERRAT, V.J. Family Hemerobiidae. In: HOFFMAN, K.M.; MEINANDER, M.; PENNY, N.D.; STANGE, L.A. A guide to the lacewings (Neuroptera) of Costa Rica. California: Proceedings of the California Academy of Sciences, 2002. v.53. n.12, p.238-251.

NAPOLEÃO, G. Subsídios para história de Barroso. Barbacena: Folha de Viçosa, 1979. 123p. 